\title{
Limitations in advance task preparation: Switching the relevant stimulus dimension in speeded same-different comparisons
}

\author{
NACHSHON MEIRAN and HADAS MARCIANO \\ Ben-Gurion University of the Negev, Beer-Sheva, Israel
}

\begin{abstract}
When participants switch between relevant stimulus dimensions in speeded classification tasks, taskswitching cost is reduced by advance preparation. Previous studies in which speeded classificationtasks were used have suggested that this effectresults from attending to the relevant stimulus dimension. Because selective attention to the relevant stimulus dimension in same-different judgments is relatively poor (e.g., Santee \& Egeth, 1980), it was predicted that advance task preparation for a shift in the relevant stimulus dimension would be compromised. This prediction was borne out in two experiments comparing dimension shifts (shape vs. fill) with task rule shifts (same? vs. different?) and shifts in the mapping of right-left keys to yes and no responses (yes-no vs. no-yes). The results indicate that advance attentional selection of the relevant dimension is an optional preparatory strategy in task switching, employed only in conditions enabling flexible refocusing of attention.
\end{abstract}

Typically, task switching is associated with a decrement in performance, called switching cost (e.g., Allport, Styles, \& Hsieh, 1994; Fagot, 1994; Mayr \& Keele, 2000; Monsell \& Driver, 2000; Rogers \& Monsell, 1995; but see Jersild, 1927, and Spector \& Biederman, 1976, as well as Meiran, 2000b, for examples of conditions without switching cost). Formally, switching cost is the difference in performance (especially in reaction time [RT] between two types of trials: switch trials, in which the task is different from that in the preceding trial, and nonswitch trials, in which the task is the same as that in the preceding trial.

In this literature, special attention has been given to the ability to prepare for a switch. The reason is that several theories assume that task information must be activated or specified before task execution begins (e.g., Logan \& Gordon, 2001; Norman \& Shallice, 1986). This ability has traditionally been linked to an empirical marker: the reduction in switching cost as a result of advance task preparation. This preparation effect is considered to be an important marker of flexible cognitive task control. For example, Allport et al. (1994), who found negligible reduction in switching cost owing to advance preparation, concluded that switching cost does not reflect a cognitive control operation. In contrast, Rogers and Monsell (1995) and Meiran (1996) found that advance preparation reduced switching cost and concluded that switching cost reflects the increased demand for control processing in the switch condition.

This research was supported by a research grant from the Israeli Science Foundation to the first author. We thank Alex Gotler and Roy Luria for help in programming and data analysis. Correspondence concerning this article should be addressed to N. Meiran, Department of Behavioral Sciences and Zlotowski Center for Neuroscience, Ben-Gurion University of the Negev, Beer-Sheva, Israel (e-mail: nmeiran@ bgumail.bgu. ac.il).
Despite the fact that preparation effects on switching cost have clear importance for cognitive control, relatively little is known about their underlying processes or boundary conditions, as compared with other effects in the task-switching literature. There are few related pieces of evidence. Rogers and Monsell (1995) found that advance preparation reduced switching cost only when the preparatory interval was constant for a block of trials, and not when it varied randomly. According to these authors, the blocking of the preparatory interval enabled a flexible, but riskier, strategy of advance task preparation. Gotler and Meiran (2001) used a paradigm in which tasks were ordered randomly and each trial began with a task cue. They found that removing the task cue upon the presentation of the imperative stimulus increased the effects of preparation on switching cost by increasing switching cost in the short preparatory interval. Presumably, removing the task cue forced participants to process the cue more thoroughly upon its presentation, rather than delaying preparation until after the presentation of the imperative stimulus (e.g., de Jong, 2000; Rogers \& Monsell, 1995). This resulted in a greater degree of preparation in all trials, including trial $n-1$. Therefore, performance in the following trial $n$ required a greater degree of advance preparation. Meiran, Chorev, and Sapir (2000; see also Meiran, 1996, Experiment 4) found that a single session of practice reduced the effects of advance task preparation on switching cost by reducing switching cost in the short preparation interval. An additional session of practice did not modulate advance preparation, because it reduced switching cost to the same degree regardless of whether the preparatory interval was short or long.

The final example is most relevant to the present study because it concerns a test of Meiran's (2000a) model. Before describing the experiment, we will briefly present the model. 
This model describes a particular control strategy used in those task-switching experiments that involve speeded classification tasks. It is argued that participants control their response selection by directing their selective attention to the relevant dimension in the target stimulus (see Shalev \& Algom, 2000, for recent evidence regarding the special status of attention to dimensions). Specifically, it is argued that response selection and response activation are based on an interaction between abstract mental representations of the target stimuli, as well as between the possible responses (cf. Hommel, 1997). This representation is so abstract that the codes being used for stimuli and responses are similar to one another and constitute a common representational domain (see Hommel, Müssler, Aschersleben, \& Prinz, in press, for a review of supporting evidence). There is one notable asymmetry in this strategy: Selective attention does not filter out irrelevant response information, although it filters out irrelevant stimulus information. Accordingly, the responses become roughly equally associated with their two possible interpretations. Thus, for example, if the experiment involves switching between size classification (small-large) and shape classification (circle-square), the keypress indicating either circle or small, depending on which task is currently being executed, is represented by both of these attributes, with more or less equal weights being given to them. The same is true for the key used to indicate either square or large. In contrast to this unbiased/unselected representation of the responses, the representation of the target stimuli is such that the irrelevant information is (nearly perfectly) filtered out. Accordingly, a small square, for example, is mentally represented as (mostly) square in the context of the shape task and as (mostly) small in the context of the size task.

The filtering of irrelevant stimulus information is sufficient to ensure correct response selection. The reason is that once the target stimulus is represented as having (nearly) only one feature, this single feature is sufficient to map the stimulus to the correct response. For example, the feature small would map to the key representing both small and circle, and not to the otherkey, representing large and square.

A further assumption in the model is that the redirection of selective attention to the relevant stimulus dimension can be performed before the presentation of the target stimulus, provided that participants are given sufficient time for advance preparation. However, if this preparatory interval is too short, the duration required to redirect selective attention is added to the RT. Because attention refocusing is required only in switch trials, redirecting attention is reflected in the task-switching cost. Thus, according to the model, (most of) the reduction in switching cost owing to advance preparation reflects the fact that attentional refocusing took place during the preparatory interval. Somewhat counterintuitively, the model argues that switching between speeded classification tasks does not involve the retrieval of relevant stimulus-response mappings. This aspect of the model is required in order to explain the fact that preparation does not reduce task con- gruity effects (see Meiran, 2000a, 2000b, for a review of the relevant literature and a list of replicable effects that the model explains).

To return to Meiran (2000b), this experiment tested the following prediction. Given Meiran's (2000b) model, efficient response selection can be achieved without the refocusing of attentional selection, provided that the target stimuli do not contain irrelevant information, so that their single attribute can directly map to the correct keypress. The experiment involved switching between two tasks. The tasks involved the location of a target stimulus within a $2 \times 2$ grid. The two tasks were $u p-d o w n$ and right-left (Meiran, 1996). In this particular experiment, there were two types of target stimuli, randomly intermixed. One type was bivalent, containing information relevant to both tasks (e.g., a target positioned in the upper-left corner of the $2 \times 2$ grid, which therefore contained both $u p$ and left information). Another type of target stimulus was univalent, containing information related to the current task only (e.g., a target positioned in the upper part of the grid, yet centered horizontally - thus, one that could only be classified as $u p$ ). As was predicted, the task-switching cost was large and was reduced by advance preparation when the target stimuli were bivalent, as in previous studies. Presumably, the redirection of selective attention was required to filter out the irrelevant information in these stimuli. In contrast, the switching cost was much smaller and was barely affected by advance preparation when the target stimuli were univalent and attentional refocusing was not required for successful response selection. Presumably, even when the preparatory interval was short, the participants could proceed directly to response selection without needing to first redirect their attention to the relevant dimension.

Note that Meiran's (2000a) model describes a particular control strategy. This strategy is probably chosen in response to the constraints afforded in the particular experimental setting. In this setting, selectively attending to the relevant target stimulus dimension is probably the easiest and least taxing mode of control. However, the strategy is likely to change in conditions that make selective attention difficult and taxing. This possibility was examined in the present experiments. Accordingly, we explored the ability to prepare for a task switch in same-different judgments, in which the dimension along which sameness was determined was changed between trials. Specifically, the participants switched between classifying sameness according to either shape or fill.

Previous studies have shown that, relative to speeded classification, same-different judgments are characterized by inefficiency in filtering irrelevant perceptual dimensions (e.g., Santee \& Egeth, 1980; but see also Miller, 1978, Miller \& Bauer, 1981, the references in Santee \& Egeth's study, and also Watanabe, 1988, who found evidence for efficient filtering of irrelevant information, but only under relatively restricted circumstances). In Santee and Egeth's study, participants were asked to classify figures or to judge the sameness of pairs of figures according to their shape. Critically, these researchers compared a condition in which 
the stimuli varied only in shape with conditions in which they also varied orthogonally on the irrelevant dimensions of size and shading, thus requiring filtering of irrelevant information. The results indicated no interference from the irrelevant dimensions in the classification task. Specifically, RT was statistically the same for the filtering condition and the condition involving unidimensional variation. In contrast, there was interference in the same-different task. Given these differences between tasks, it was predicted that the participants' ability to prepare for dimensional selection in same-different tasks would be compromised. Operationally, this would be reflected in a reduction or even an elimination of preparation effects on switching cost.

We also wished to rule out the possibility that this reduced preparation effect does not reflect a general property of the same-different task or a lack of motivation to prepare (de Jong, 2000). Therefore, we compared the dimension shift condition with conditions in which task aspects other than the relevant stimulus dimension were switched. For this reason, we will first provide a short review of the literature on preparation for a task switch as a function of the switched operation.

\section{Advance Task Preparation as a Function of the Switched Operation}

In almost all of the articles we reviewed, we found evidence that the preparation for a switch reduced switching cost. This was true for a change in stimulus modality (Quinlan \& Hill, 1999; but see also Spence \& Driver, 1997), a change in stimulus-response mapping between compatible mapping (left $\rightarrow$ left, right $\rightarrow$ right) and incompatible mapping $($ left $\rightarrow$ right, right $\rightarrow$ left; de Jong, 1995), and task switching (e.g., Allport et al., 1994; de Jong, 2000; de Jong, Berendsen, \& Cools, 1999; Fagot, 1994; Goschke, 2000; Mayr \& Keele, 2000; Meiran, 1996, 2000a, 2000b; Meiran et al., 2000).

We found only two exceptions for which preparation did not reduce switching cost. Both referred to switching perceptual operations. First, Los (1999a, 1999b) asked participants to name digits that were visually degraded in two different ways: added visual noise or segment deletion. Presumably, these two forms of degradation involve different perceptual operations. Los found that shifting between the two kinds of degraded digits (hence, perceptual operations) resulted in a switching cost. Nonetheless, informing participants at the beginning of the trial regarding the type of degradation that would appear in the upcoming digit did not reduce this switching cost (Los, 1999b). Second, Lamb and his colleagues (Lamb, London, Pond, \& Whitt, 1998; Lamb, Pond, \& Zahir, 2000) studied shifting between levels of hierarchical structure-for example, a large $A$ composed of small $S$ s (Navon, 1977). Participants were asked to detect an $H$ or an $S$ that appeared at either the local level or the global level of the structure. They found a cost for level switching (cf. Ward, 1982). Nonetheless, this cost was affected neither by block-wide expectancy (Lamb et al., 1998) nor by expectancy resulting from a cue at the beginning of the trial (Lamb et al., 2000).

\section{The Present Experiments}

In the present experiments, we compared three types of operation shifts within the same-different paradigm in which two stimuli were presented simultaneously and participants were required to indicate whether they were the same or different. The most important condition for our prediction involved dimension shift ( fill vs. shape). This condition was compared with two control conditions including task rule shift (same? vs. different?), and responsemapping shift (assignment of the right and left keys as yes-no, respectively, vs. no-yes, respectively). In all cases, shifts were randomly ordered, with a shifting probability of .5. It should be noted that, formally, task rule shift and response-mapping shift are equivalent, because both result in the same change in the correct overt response. However, we will show evidence that despite this formal equivalence, the participants did not treat the two conditions equally. Most important, the difference or lack of difference between response-mapping shift and task rule shift is not crucial for our main prediction concerning dimension shift.

It should be further noted that, to be able to conduct our examination, we needed to ensure that there would be a switching cost in the same-different judgments. Fortunately, two studies have shown this already. Proctor and Fisicaro (1977) presented participants with two stimuli, which were two stripes varying in color, two circles varying in size, or two letters varying in letter identity. RTs were longer when the three classes of stimuli were intermixed in the same block of trials, as compared with when only one class of stimuli was presented in a given block of trials. More recently, Garcia-Ogueta (1993) presented participants with two shapes, presented either simultaneously or sequentially, and found that mixing trials with simultaneous and sequential presentation modes resulted in a performance cost. Nonetheless, in neither of these studies were the effects of advance preparation on switching cost examined (although Proctor \& Fisicaro, 1977, studied the time course of resource demand, which may be related to advance preparation).

The other aspect of our study involves comparing switch types within an experiment. Such comparisons have been reported by previous investigators (e.g., Allport et al., 1994; Hübner, Futterer, \& Steinhauser, 2001; Kleinsorge \& Heuer, 1999; see also Van-Duren \& Sanders, 1988), but none of these experiments had findings that were relevant to our predictions concerning differential effects of preparation. With respect to preparation, we employed the common procedure, which is based on randomly ordering the trials involving the two tasks, providing a task cue at the beginning of a trial, and manipulating the interval between the task cue and the imperative stimulus, or the cue-target interval (CTI). In this procedure, the effect of advance preparation on switching cost is indexed by the two-way interaction between task switch and CTI. Accordingly, we predicted a triple interaction whereby this two-way interaction would be modulated by switch-type.

In our experiments, we presented participants with two figures, one on the right side and one on the left side of a 


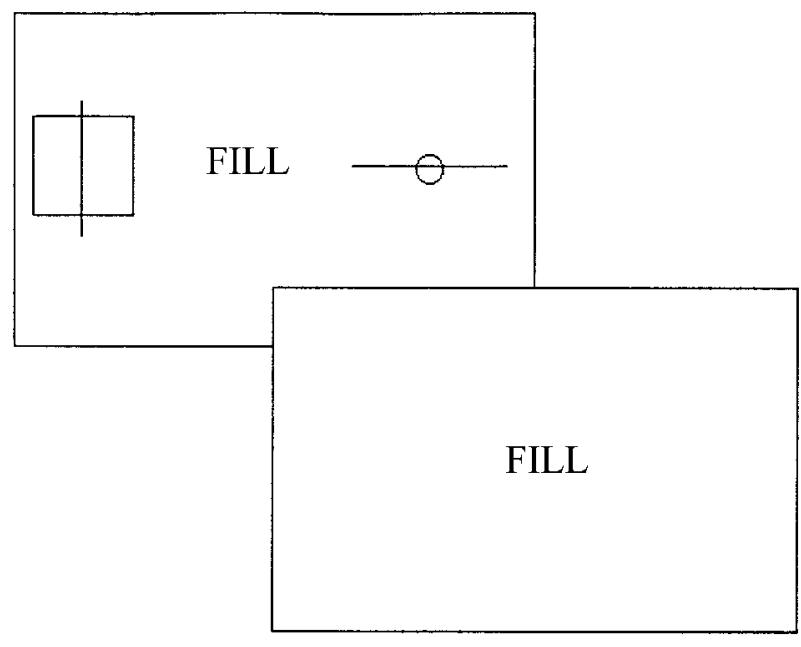

Figure 1. A schematic representation of an exemplar trial beginning with a task cue and followed by a target display. In this example, the correct answer was yes, because both figures were empty.

computer screen. The figures differed along four perceptual dimensions. They were circles or squares (shape), which were small or large (size) and full or empty (fill), and were crossed by a vertical line or a horizontal line (tilt; see Figure 1 for an example). Meiran, Hommel, Bibi, and Lev (2002, Experiment 3), who employed speeded classification tasks, used the same target stimuli as those used in the present experiments. Each of the four classification tasks studied by Meiran, Hommel, et al. (2002) involved unidimensional classification (e.g., full vs. empty) of a centrally presented figure, which could vary along four perceptual dimensions. They found that switching tasks was associated with a substantial switching cost $(140 \mathrm{msec}$, on average, when minimal time was allowed for advance preparation-i.e., the shortest CTI), which was reduced to about a sixth of its original size ( $23 \mathrm{msec}$, on average) by allowing a long CTI for advance preparation. Meiran, Hommel, et al.'s (2002) findings were relevant for the present experiments in that they showed that there is nothing special about the target stimuli used in the present experiments that would prevent advance task preparation from taking place (it should be noted that Meiran, Hommel, et al.'s [2002] results were replicated by Meiran, Neulinger, \& Mayr [2002], using the same type of task cues as those used here).

\section{EXPERIMENT 1}

Because we were interested in judgments made on the basis of a single perceptual dimension, we tried to lead the participants to adopt an analytic strategy and to prevent their making holistic same comparisons (fast same responses, if the stimuli are identical to one another in every respect). For this reason, we used the disjunctive version of the same-different task (see Farell, 1985, for a review).
Accordingly, although the two figures varied on four dimensions, shape, fill, size, and tilt, only shape and fill could be relevant; size and tilt were never relevant. Thus, the two figures were to be judged as same if they were the same with respect to the task-relevant stimulus dimension (e.g., they were judged as same if the relevant dimension was fill and both figures were empty; see Figure 1) although they differed along all of the other three irrelevant dimensions (e.g., size, shape, and tilt). In other words, different figures differed along all four dimensions, whereas same figures differed on all but one dimension.

The participants performed either the same task ("are the figures the same?") or the different task ("are the figures different?"). In the dimension shift group, the participants shifted between relevant perceptual dimensions. The relevant dimensions changed randomly from trial to trial, and each trial began with a verbal cue, instructing the participants as to which dimension was relevant, shape or fill. For any given participant in this group, the task (same, different) and response mapping were constant, and the only element that varied randomly between trials involved the relevant dimension. In the decision rule shift group, the participants shifted between the same task and the different task. The instructional cues were the words same? and different?. For any given participant, the relevant stimulus dimension and response mapping were constant. Finally, in the response-mapping shift group, the participants shifted between two possible response mappings. In one mapping, the key on the right indicated yes, and the key on the left indicated no. The instructional cue for this mapping was no-yes. For any given participant in this group, the relevant stimulus dimension and the task remained constant.

\section{Method}

Participants. Forty-eight students from Ben-Gurion University of the Negev, Israel, participated as a part of an introductory course requirement. The 16 participants within each switch type group were assigned to one of four subgroups, so that within each subgroup, the nonswitched aspects were constant. For example, when response mappings shifted, there were four subgroups of participants. For one subgroup, the task was same, and the relevant dimension was fill; for another subgroup, the task was different, and the relevant dimension was fill. Two additional subgroups performed the shape task. The participants were assigned to the various conditions according to order of entry into the experiment.

Stimuli. We used IBM-PC compatible microcomputers controlled by software written in MEL language (Schneider, 1988). Target stimuli were presented in white on a black background and varied along four dimensions. The stimulus was either a small/large circle (with a diameter subtending a visual angle of approximately $1.4^{\circ}$ or $3.0^{\circ}$ ) or a small/large square (each side subtending $1.4^{\circ}$ or $3.0^{\circ}$ ) that was either empty (only the circumference depicted in white on black) or filled (the entire figure filled with a light gray color). A line that subtended $4.5^{\circ}$ crossed the figure in its middle and was either horizontal or vertical. One stimulus was presented on the right side of the screen, and the other was presented on the left, their centers $9.8^{\circ}$ from each other. The instructional cues were the Hebrew equivalents of fill, shape, same?, different?, yes-no, and no-yes. They were positioned in the center, between the figures. The height of the words was $0.5^{\circ}$, and the width ranged between $1.9^{\circ}$ and $3.3^{\circ}$. The two fig- 
ures either were completely different along all four dimensions or were identical along the task-relevant dimension only and different on all other dimensions.

Procedure. The participants were tested in two identical sessions, separated by 1-3 days. Each session comprised 25 warm-up trials followed by four identical blocks of 100 trials. In each trial, the CTI $(170,470,1,470$, or $2,970 \mathrm{msec})$, target stimuli, and relevant rule changed randomly with equal probability. The relevant rule depended on switch type. Each trial consisted of presenting the instructional cue for a variable CTI, followed by the presentation of the figures along with the instructional cue until the response. After the response was given, the screen went blank for 1,430 msec. Previous studies had indicated that switching cost was reduced by increasing the response-cue interval (e.g., Meiran et al., 2000). Although we did not perform a preliminary experiment in which the response-cue interval varied, experience with the up-down/right-left paradigm indicated that a response-cue interval of less than $1 \mathrm{sec}$ was sufficient for most of the reduction in switching cost. Thus, with an interval of $1,430 \mathrm{msec}$, we assumed that nearly all of the CTI-related reduction in switching cost reflected advance reconfiguration, rather than the passive dissipation of the task set that was adopted in trial $n-1$.

\section{Results and Discussion}

The first trial in a block and trials preceded by errors or by exceedingly long RTs (3,000 msec) were omitted from all analyses. Trials in which the RT was exceedingly long $(3,000 \mathrm{msec})$ were analyzed for accuracy, but not for RT. We computed the mean RT per condition and submitted these means to various analyses of variance (ANOVAs).

Preparation effects on switching cost. We conducted a $3 \times 2 \times 4$ mixed model ANOVA with the betweensubjects independent variable switch type (dimension, decision rule, or response mapping) and the within-subjects independent variables switch (switch vs. nonswitch) and CTI $(170,470,1,470$, or 2,970 msec). To save space, we will not report effects that were qualified by higher order interactions. Alpha level was set at .05 in all analyses.

The triple interaction was statistically significant $\left[F(6,135)=3.12, M S_{\mathrm{e}}=1,909\right]$, indicating differences between conditions with respect to the effects of preparation on switching cost (Figure 2). To show the source of this triple interaction, we examined the simple interactions between CTI and switch within each switch type. This simple interaction was statistically significant in the decision rule group $\left[F(3,135)=11.86, M S_{\mathrm{e}}=1,909\right]$ and in the response-mapping group $\left[F(3,135)=4.45, M S_{\mathrm{e}}=1,909\right]$ (indicating that switching cost was reduced by preparation), but not in the dimension group $(F<1)$. Because the lack of significance could reflect low statistical power, we increased the power by examining the one degree-offreedom linear component of the simple interaction in the dimension switch group, and it was nonsignificant as well $(F<1)$.

Given the fact that the effect of CTI on switching cost, when found, indicated cost reduction, we examined the triple interaction between switch, switch type, and the linear component of CTI. Although the function relating CTI to switching cost is generally asymptotic, rather than linear (e.g., Meiran et al., 2000), visual inspection suggested that, in the present experiment, the asymptote had not been reached and switching cost was reduced at a more or less constant rate with increasing CTI. In agreement with visual inspection, this test was significant $[F(2,45)=$ $\left.5.49, M S_{\mathrm{e}}=2,539\right]$, whereas the deviation from linearity was nonsignificant $(F=1.23)$. Notably, the linear component of the interaction was also significant when the nonswitch condition was represented by the first task repetition, excluding higher order repetitions $[F(2,45)=$ $\left.3.37, M S_{\mathrm{e}}=3,168\right]$, with a nonsignificant residual interaction $(F<1)$. These analyses show that the three switch types differed with respect to the linear rate of cost reduction, with two groups showing such a reduction, whereas one group did not.

We also conducted a three-way ANOVA on proportions of errors (bottom of Figure 2) according to group, CTI, and switch and found that the triple interaction approached significance $\left[F(6,135)=2.01, M S_{\mathrm{e}}=0.0002, p=.068\right]$, indicating a similar, albeit noisier, version of the pattern found for RT. Thus, the findings argue against a speedaccuracy account.

Nonswitch RT. Although the present experiments concentrated on switching cost, some conclusions can also be drawn with respect to mixing cost. Mixing cost is defined as the difference in performance (e.g., RT) between the nonswitch condition and a condition involving a single task (Fagot, 1994; Logan \& Gordon's, 2001, concurrence cost; Meiran et al., 2000; see also Kray \& Lindenberger, 2000; Los, 1996). The reason one may draw conclusions regarding mixing cost is that, if the single-task baseline had been included, it would have been the same for all of the switch types we studied. Specifically, this single-task baseline would have involved a constant dimension, a constant task rule, and a constant response mapping. Given the definition of mixing cost, differences in nonswitch RTs among the three switch types would indicate differences in mixing cost, despite the fact that the absolute size of mixing cost was not determined. Another relevant piece of evidence concerning mixing cost, to be discussed in the Task Repetition Effects section below, is whether performance improves as a consequence of repeating the same task over and over. If task repetition continues to be effective beyond the first repetition, this suggests that mixing cost is larger than zero, because the single-task baseline essentially comprises consistent task repetitions.

The overall comparison between groups was significant $\left[F(2,45)=5.16, M S_{\mathrm{e}}=228,395\right]$. Follow-up pairwise comparisons indicated that dimension shift RTs were faster than rule shift RTs $\left[F(1,45)=4.16, M S_{\mathrm{e}}=228,395\right]$ and key shift RTs $\left(F(1.45)=10.06, M S_{\mathrm{e}}=228,395\right)$, which did not differ significantly from one another $(F=$ 1.28). These results indicated that the smallest mixing cost was found in the dimension shift condition.

Rule shift versus response-mapping shift. The rule shift condition and the key-mapping shift condition were included mainly for the purpose of comparison, and as such, any differences between them were not critical to the present study. Formally, these conditions were equivalent, because the consequences on overt responses were the same for the two types of switch. Specifically, changing the 

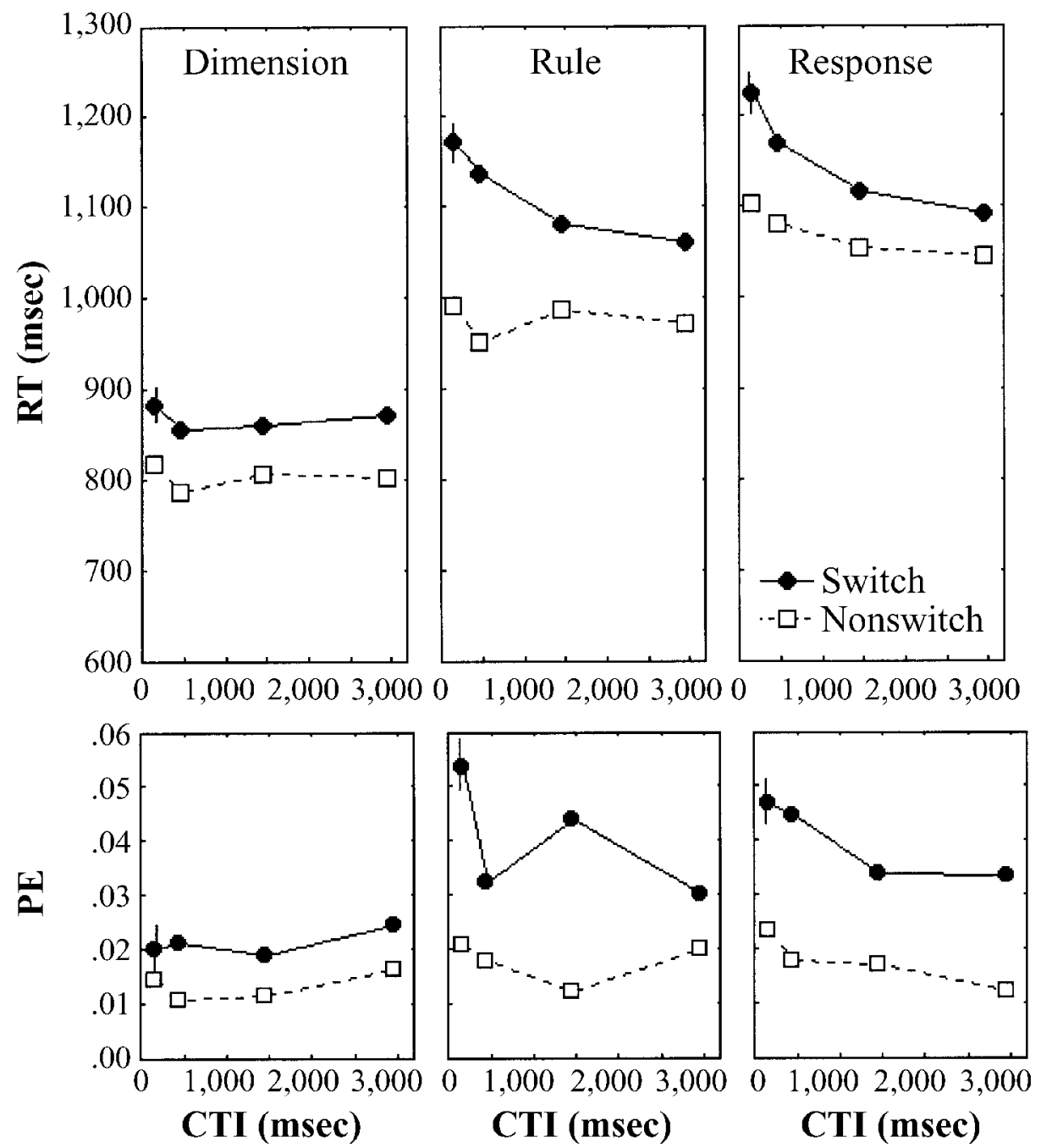

Figure 2. Reaction time (RT, in milliseconds) and proportion of errors (PE) as a function of switch type and cue-target interval (CTI, in milliseconds) in Experiment 1. The uniform $95 \%$ confidence interval for all means is presented once to prevent visual noise. It is useful for evaluating reductions in switching cost owing to increasing CTI, and based on the $M S_{\mathrm{e}}$ of the triple interaction, $\pm 13 \mathrm{msec}$ and \pm .004 PE.

rule from same? to different? reversed the yes-no decision. Moreover, each of the participants in that group received only one mapping, either yes-no or no-yes. Therefore, a rule change was equivalent (for overt responses) to changing the mapping from, for example, yes-no to no-yes. Nonetheless, this formal equivalence does not necessarily imply that the conditions were psychologically equivalent. In fact, the results indicated a significant difference in switching cost between them, as can be seen in Figure 2 $\left[F(1,45)=5.26, M S_{\mathrm{e}}=10,041\right]$. This result suggests that the participants did not treat the two conditions equally.

Was there a shift in the dimension shift condition? Importantly, in the dimension shift group only, the participants could, in principle, ignore the relevant dimension altogether and simply judge whether the two targets dif- fered on all dimensions $(=$ different $)$ or were the same along a single dimension (= same). Such a strategy is predicted to result in zero switching cost in different responses, in which the relevant dimension does not matter. However, it is conceivable that the strategy would produce a switching cost in same responses, for which a given dimension must be attended. To explore this possibility, we conducted an ANOVA in the dimension shift group only, including the independent variables decision (same vs. different), CTI, and switch. There was a significant 57msec switching cost for different judgments (911 vs. $854 \mathrm{msec}$ ), which did not differ reliably from the significant 72 -msec cost for same judgments ( $826 \mathrm{vs} .754 \mathrm{msec}$; $F=1.49$ ). Thus, the results did not support the possibility that the participants simply looked for a single same di- 
mension. Additional evidence against this possibility is the fact that nonswitch RTs were shortest in the dimension shift group. However, if the participants had checked all the stimulus dimensions, as this strategy suggests, this should have slowed them, relative to the other groups for which only one dimension was to be checked (we thank Sander Los for pointing out this to us).

Another variant of the single-task argument just described is that the source of switching cost in the dimension shift group reflects the fact that the participants searched all of the dimensions on every trial. According to this explanation, switching affected the order in which the dimensions were searched. The results rule out this possibility as well, for the following reason. If a serial selfterminating search is assumed, such a strategy is predicted to result in switching cost in same judgments, because the hypothesis is that the dimension of sameness would be the first to be searched in nonswitch trials. However, because different responses require searching all the dimensions, there should not be a switching cost in such responses. For this very reason, exhaustive search is not predicted to result in a switching cost in either response. Because we observed switching costs in both same and different responses, this alternative explanation is ruled out as well.

The fact that different responses were slower than same responses $\left[F(1,45)=20.75, M S_{\mathrm{e}}=26,302\right]$ is the common finding in disjunctive same-different tasks (e.g., Farell, 1985), which provides some validation that strategies were invoked in dealing with the same-different task that were similar to those found in previous studies in which there was no switching. Taken together, the present analysis indicates that the participants treated the dimension switch as a task switch.

Task repetition effects. As was mentioned before in the Nonswitch RT section, comparing the groups with respect to their mean nonswitch RTs indicated a difference in mixing cost. However, we wished to demonstrate that there was a mixing cost in all three groups. We therefore decided to examine whether consecutive task repetitions would produce performance gain (Meiran et al., 2000). Given the fact that a single-task condition would have involved more task repetitions than would mixed-task conditions, such a result would indicate that performance in the nonswitch condition did not reach that in the single-task condition. In other words, this would imply that mixing cost was positive. We therefore analyzed the results according to group, CTI, and consecutive task repetition, while excluding switch RT to avoid repeating the preceding analyses. Thus, in consecutive task repetition, we compared the first, second, third, and fourth consecutive task repetitions (which, in Rogers $\&$ Monsell's, 1995, terms are the second through fifth positions in the run, because in these terms, the first position in the run indicates switch trials). To avoid repeating previous results, we concentrated only on effects involving consecutive task repetition. This variable was associated with a significant main effect $\left[F(3,135)=7.22, M S_{\mathrm{e}}=\right.$ $7,235]$ and did not interact significantly with group. The linear component of this main effect was highly significant $\left[F(1,45)=15.67, M S_{\mathrm{e}}=9,389\right]$, indicating an average reduction of $12 \mathrm{msec}$ per each task repetition, whereas the deviation from linearity was clearly nonsignificant $(F<1$; see Figure 3). Importantly, the linear component did not differ significantly between groups $(F<1)$. These results lead to two conclusions. First, because the singletask baseline was based on more task repetitions than just four, it would be reasonable to conclude that mixing cost was positive in all groups. Second, the difference between groups in mixing cost was unlikely to be due to differential task repetition effects, because these effects were comparable in size in the present analysis, as was indicated by a nonsignificant two-way interaction.

\section{EXPERIMENT 2}

The goal of Experiment 2 was to replicate and extend the finding concerning reduced effects of preparation on switching cost in the dimension shift condition. Such a replication seems essential, given the null result in this condition. To increase the generality of the conclusions, two conditions were compared between groups in this experiment. The simultaneous presentation condition was a straightforward replication of the dimension shift condition in Experiment 1 . It was compared with a sequential presentation condition, in which, after the task cue was provided, one of the two randomly chosen targets was presented for $300 \mathrm{msec}$ before the entire display was presented, including both targets and the task cue. The fact that GarciaOgueta (1993) found that switching between simultaneous and sequential presentation resulted in a cost suggests that these presentation modes invoke different processing strategies. Moreover, Santee and Egeth (1980, Experiment 3) also examined selection in sequentially presented stimuli and found less interference from the size dimension to the shape dimension as the interstimulus interval increased. On the basis of the reasoning so far, this may suggest an improvement in participants' ability to efficiently direct attention to the relevant stimulus dimension. Accordingly, our aim was to explore the possibility that preparation-related reduction in switching cost would be found with sequential presentation, but not with simultaneous presentation.

\section{Method}

Participants. The 24 participants came from the same population as did those who took part in Experiment 1. Half of them were assigned to each of the two presentation mode groups.

Stimuli and Procedure. The only difference, relative to Experiment 1 , was that, for the sequential presentation group, one of the two targets (half of the time the right target, and half the left target) was presented first, and after $300 \mathrm{msec}$ the second target was added to the display. In that group, RT was measured from the presentation of the second target until the response.

\section{Results and Discussion}

The results were treated as in Experiment 1, and cell means were analyzed according to presentation mode (si- 

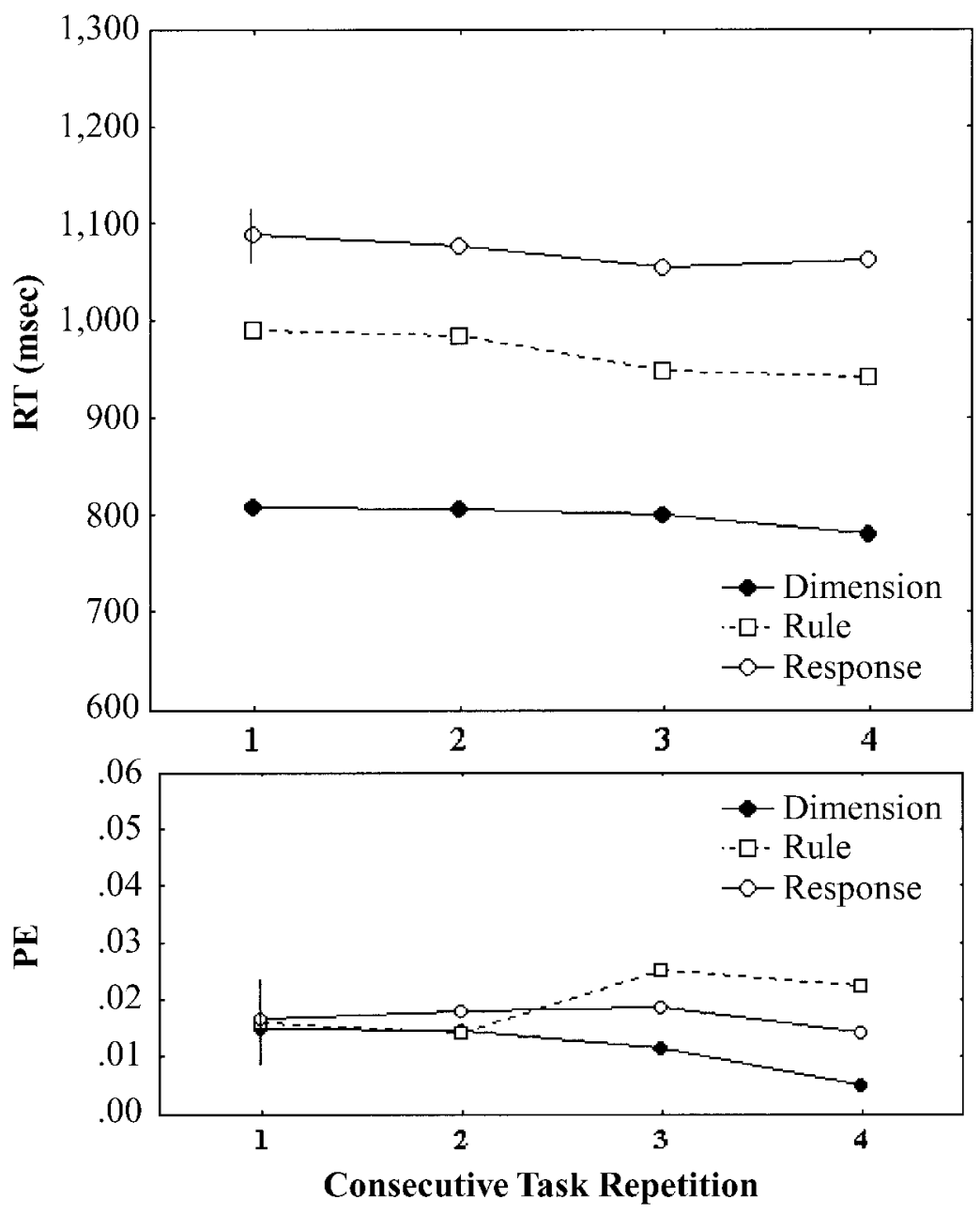

Figure 3. Reaction time (RT, in milliseconds) and proportion of errors (PE) as a function of switch type and consecutive task repetition in Experiment 1. The uniform 95\% confidence interval is useful for evaluating reductions in RT owing to consecutive task repetitions, and based on the $M S_{\mathrm{e}}$ of the (nonsignificant) two-way interaction between consecutive task repetition and switch type, $\pm 25 \mathrm{msec}$ and $\pm .01 \mathrm{PE}$.

multaneous vs. sequential), CTI, and switch (see Figure 4). Only two main effects were significant. The main effect of presentation mode $\left[F(1,22)=9.88, M S_{\mathrm{e}}=1,600,797\right]$ indicated faster responses in sequential presentation $(706 \mathrm{msec})$ than in simultaneous presentation $(993 \mathrm{msec})$. This result indicates that the participants took advantage of the sequential presentation and processed the first target before the second target appeared (see Santee \& Egeth, 1980 , for a similar result). In fact, the benefit $(287 \mathrm{msec})$ was almost as large as the time allowed for inspecting the first target, before the entire display came up (300 msec). The second significant effect was that of switch $[F(1,22)=$ $\left.108.72, M S_{\mathrm{e}}=7,901\right]$, indicating longer RTs in switch trials $(883 \mathrm{msec})$ than in nonswitch trials $(816 \mathrm{msec})$. Although the main effect of CTI approached statistical significance $\left[F(3,66)=2.35, M S_{\mathrm{e}}=19,485, p=.08 ; 841\right.$, 832,863 , and $863 \mathrm{msec}$, in the shortest through longest CTIs, respectively], none of the other sources of variance approached significance - most noteworthy, including interactions involving CTI and task switch (all $F_{\mathrm{S}}<1$ ).

The results were not as predicted, since the trend of means indicated some reduction in switching cost in the simultaneous group. However, this trend was quite different from the trend that indexes preparation effects on switching cost. Specifically, these effects are characterized by an overadditive interaction between task switch and CTI, whereby the effects of CTI are larger in switch trials than in nonswitch trials. The nonsignificant interaction that we observed here was underadditive: Whereas CTI did not have an effect in switch trials, increasing CTI in nonswitch trials resulted in slowing. Similar underadditive interactions usually index the dissipation of the task set adopted in the preceding trial, rather than set preparation. The reasoning behind this interpretation is that nonswitch trials require the same task set as that which had been adopted in the preceding trial. If this set dissipates, this re- 

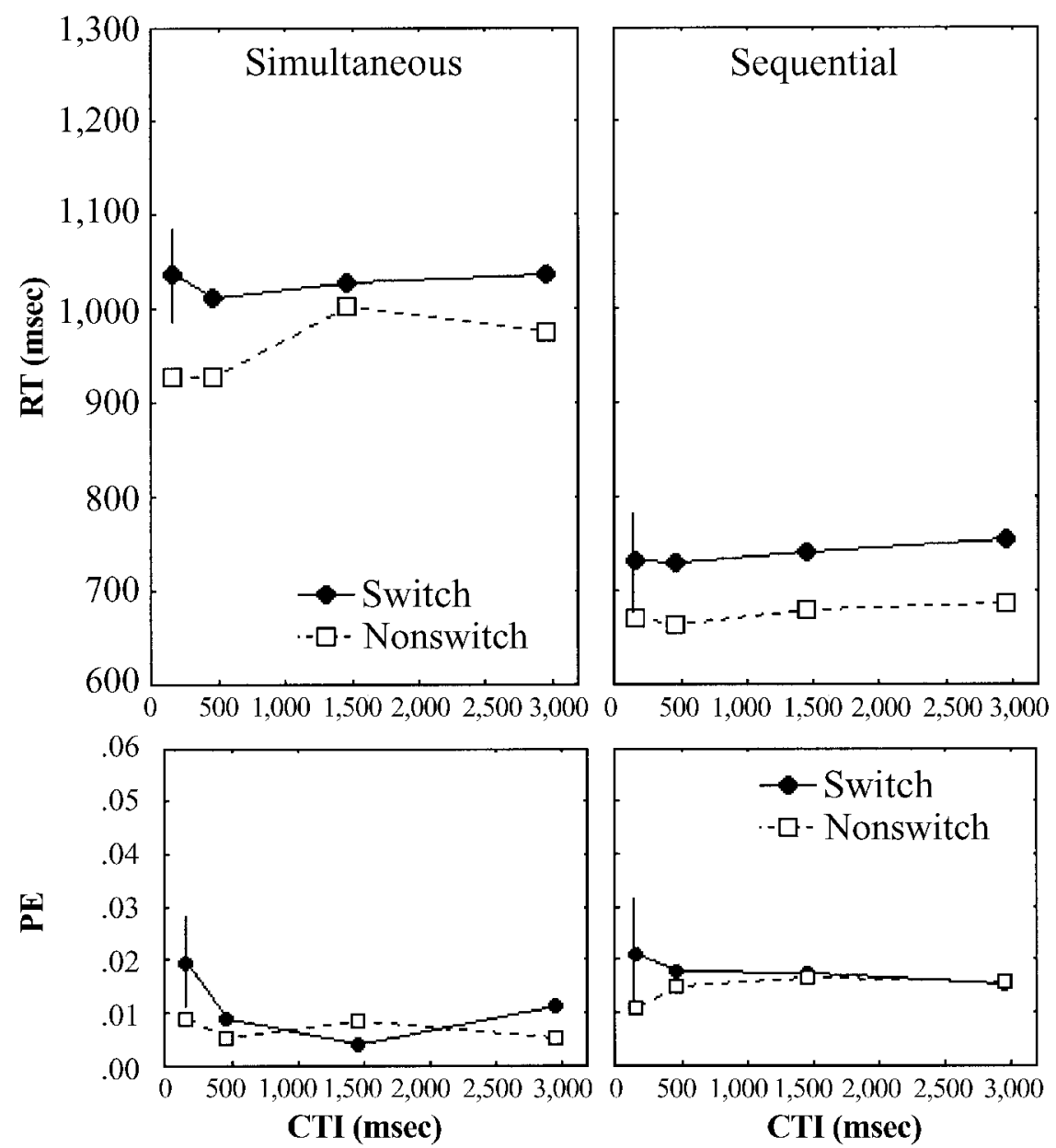

Figure 4. Reaction time (RT, in milliseconds) and proportion of errors (PE) as a function of presentation mode and cue-target interval (CTI, in milliseconds) in Experiment 2. In the sequential presentation, RT was measured from the onset of the second target. The uniform $95 \%$ confidence interval is useful for evaluating reductions in switching cost owing to increasing CTI, and based on the $M S_{\mathrm{e}}$ of the (nonsignificant) triple interaction, $\pm 55 \mathrm{msec}$ and \pm .01 PE.

sults in a lesser degree of readiness in nonswitch trials (see Meiran et al., 2000). Accordingly, the fact that this underadditive trend was restricted to the simultaneous condition may be attributed to the fact that, in the sequential condition, RT measurement began 300 msec later (after the presentation of the second target). This allowed more complete dissipation of the task set adopted in the preceding trial.

In a parallel analysis on the proportion of errors (Figure 4, bottom), we found only a significant main effect of task switch $\left[F(1,22)=5.41, M S_{\mathrm{e}}=0.0004\right]$, in the same direction as that found for RT. Thus, the results argue against a speed-accuracy account.

\section{GENERAL DISCUSSION}

Previous research on task switching in which speeded classification tasks were used led to a series of empirical regularities, which have been reasonably successfully ex- plained by Meiran's (2000a) model. This model was supported both by model fitting and, most importantly, by testing some of its novel predictions (Meiran, 2000b). One of its crucial aspects is the suggestion that the reduction in switching cost owing to advance preparation reflects the redirection of selective attention to the relevant stimulus dimension. This choice of control strategy probably results from the relative ease in drawing selective attention to the relevant stimulus dimension in speeded classification tasks.

Conditions in which such drawing of attention becomes difficult and taxing were therefore predicted to result in a change in control strategy. In the present study, we examined such a condition by capitalizing on the known differences between speeded classification tasks, in which selective attention to the relevant stimulus dimension is relatively efficient, and speeded same-different judgments, for which such selection is far less efficient (e.g., Santee \& Egeth, 1980). Accordingly, we predicted that the advance prepa- 
ration for a dimension switch would be compromised in same-different judgments. The results of the two experiments supported this prediction.

We can rule out several alternative explanations of these results. Specifically, there is nothing special about switching dimensions in the present set of stimuli that prevents advance task preparation. The reason is that other studies in which dimension switching in speeded classification was explored and the same stimuli were used found large reductions in switching cost owing to advance preparation (Meiran, Hommel, et al., 2002; Meiran, Neulinger, \& Mayr, 2002). In addition, the lack of advance preparation cannot be attributed to lack of motivation to prepare (de Jong, 2000) or to a general property whereby advance task preparation is not possible in same-different judgments. This is because preparation resulted in a reduction in switching cost in the conditions involving rule shift or responsemapping shift.

An interesting question is how switching was possible at all, given the limitation on selective attention, and why switching dimensions produced cost. With respect to the first part of the question, although the literature has suggested that there are limitations on selective attention in same-different judgments, these limitations were not complete, because the participants could perform the instructed task. We suggest that, given the relative difficulty of drawing attention to the relevant dimension in same-different judgments, the participants chose to postpone the direction of attention until after the target stimuli had been presented. Rogers and Monsell (1995) called this control strategy stimulus-cued reconfiguration. It implies that the empty CTI was not used for advance preparation and, thus, its increase did not lead to performance gain. Hence, according to this reasoning, part of the switching cost we observed reflected the time it took to direct attention to the relevant stimulus dimension after the target stimulus had been presented.

Stimulus-cued reconfigurationis unlikely to fully explain the switching cost, because task repetitions led to faster responses. This, in itself, could contribute to switching cost because, by definition, switching cost reflects the difference between the first and second times in which the task has been performed. Nonetheless, the observed slope was $12 \mathrm{msec}$ per task repetition, whereas the switching cost in the dimension shift group (Experiment 1) was $868-809=$ $59 \mathrm{msec}$ even after excluding high-order repetitions. In other words, we suggest that the switching cost we observed has at least two components: one owing to task repetition (micro-practice, Rogers \& Monsell, 1995; retroactive adjustment, Meiran, 1996) and another owing to stimuluscued reconfiguration.

The broader implications of the present results are mainly two. First, it seems that the notion of task switching is relatively crude and should thus be replaced by finer and more precise terms. For example, the literature has suggested that switching cost increases with an increasing number of switched task elements (e.g., Hübner et al., 2001; Mayr, 2001; but see also Allport et al., 1994, for a null difference, and Logan \& Gordon, 2001, for a theoretical treat- ment). These results argue against the notion of a unitary task set that is activated as a single package. They are more consistent with a distributed notion of a task set, according to which the various task aspects may be activated separately or as a unit, depending on the structure of the experiment (e.g., Hübner et al., 2001; but see also Logan \& Gordon, 2001). If this reasoning is correct, the issue concerning which instances qualify as a task switch - that is, which elements were switched and how many of thembecomes rather arbitrary and a matter of theoretical taste.

The present study (also Meiran, 2000b) indicates an additional qualification. It seems the various shift task elements might be shifted at different points in time. For example, in Experiment 1, switching was associated with a shift of a single task element in all three groups, yet the results depended on which element had been switched. When the switch was on the relevant dimension, the participants delayed focusing of attention until after the presentation of the target stimuli (stimulus-cued reconfiguration). When the task rule or response mapping was involved, the elements were at least partly switched in preparation for the target stimulus during the empty CTI.

The second broad implication concerns the attempt to identify a general model for cognitive control in task switching. This approach has characterized most of the previous studies on task switching, in which a control strategy that had been identified in one experimental setup was tested in another setup. For example, Allport et al. (1994) studied switching between color naming and word reading and used Stroop stimuli. On the basis of their results, they argued that advance preparation does not reduce task-switching cost. This conclusion had been debated by Rogers and Monsell (1995) and by Meiran (1996), but neither of these studies used Stroop task switching. The comparison between Meiran, Hommel, et al.'s (2002) results and those of the present study suggests that an attempt to characterize general switching processes is unlikely to succeed. A more plausible route of inquiry is to first identify the various control strategies that participants use in task-switching experiments. At the next stage, the research should concentrate on the situational constraints and their effects on the choice of control strategies. One control strategy, which we have identified in previous research (Meiran, 2000a, 2000b), was based on the direction of attention to the relevant stimulus dimension. The present results suggest that this strategy is unlikely to be chosen in conditions in which attention to dimensions is relatively limited and, probably, its redirection is difficult and taxing. In such conditions, participants choose a relatively less taxing and more bottom-up strategy of stimulus-cued reconfiguration.

\section{REFERENCES}

Allport, D. A., Styles, E. A., \& Hsieh, S. (1994). Shifting intentional set: Exploring the dynamic control of tasks. In C. Umiltà \& M. Moscovitch (Eds.), Attention and performance XV: Conscious and nonconscious information processing (pp. 421-452). Hillsdale, NJ: Erlbaum. DE JONG, R. (1995). Strategical determinants of compatibility effects with task uncertainty. Acta Psychologica, 88, 187-207. 
DE JoNG, R. (2000). An intention-activation account of residual switch costs. In S. Monsell \& J. Driver (Eds.), Control of cognitive processes: Attention and performance XVIII (pp. 357-376). Cambridge, MA: MIT Press.

De Jong, R., Berendsen, E., \& Cools, R. (1999). Goal neglect and inhibitory limitations: Dissociable causes of interference effects in conflict situations. Acta Psychologica, 101, 379-394.

FAGOT, C. (1994). Chronometric investigations of task switching. Unpublished doctoral dissertation, University of California, San Diego.

FARELL, B. (1985). "Same"-"different" judgments: A review of current controversies in perceptual comparisons. Psychological Bulletin, 98, 419-456.

Garcia-OGueta, M. I. (1993). Internal attentional switching: Effects of predictability, complexity and practice. Acta Psychologica, 83, 13-32.

GoschKE, T. (2000). Intentional reconfiguration and involuntary persistence in task-set switching. In S. Monsell \& J. Driver (Eds.), Control of cognitive processes: Attention and performance XVIII (pp.331-356). Cambridge, MA: MIT Press.

Gotler, A., \& Meiran, N. (2001). Cognitive processes underlying a frontally-mediated component of task-switching. Brain \& Cognition, 47, 142-146.

Hommel, B. (1997). Toward an action-concept model of stimulus-response compatibility. In B. Hommel \& W. Prinz (Eds.), Theoretical issues in stimulus-response compatibility (pp. 281-320). Amsterdam: Elsevier.

Hommel, B., Müssler, J., Aschersleben, G., \& Prinz, W. (in press). The theory of event coding (TEC): A framework for perception and action planning. Behavioral \& Brain Sciences.

Hübner, R., Futterer, T., \& Steinhauser, M. (2001). On attentional control as a source of residual shift costs: Evidence from two-component task shifts. Journal of Experimental Psychology: Learning, Memory, \& Cognition, 27, 640-653.

Jersild, A. T. (1927). Mental set and shift. Archives of Psychology, 14 (Whole No. 89).

Kleinsorge, T., \& Heuer, H. (1999). Hierarchical switching in a multidimensional task space. Psychological Research, 62, 300-312.

Kray, J., \& Lindenberger, U. (2000). Adult age differences in task switching. Psychology \& Aging, 15, 126-147.

Lamb, M. R., London, B., Pond, H. M., \& Whitt, K. A. (1998). Automatic and controlled processes in the analysis of hierarchical structure. Psychological Science, 9, 14-19.

LAMb, M. R., Pond, H. M., \& ZAHIR, G. (2000). Contributions of automatic and controlled processes to the analysis of hierarchical structure. Journal of Experimental Psychology: Human Perception \& Performance, 26, 234-245.

Logan, G. D., \& Gordon, R. D. (2001). Executive control of visual attention in dual-task situations. Psychological Review, 108, 393-434.

Los, S. A. (1996). On the origin of mixing costs: Exploring information processing in pure and mixed blocks of trials. Acta Psychologica, 94, $145-188$.

Los, S. A. (1999a). Identifying stimuli of different perceptual categories in mixed blocks of trials: Evidence for cost in switching between computational processes. Journal of Experimental Psychology: Human Perception \& Performance, 25, 3-23.

Los, S. A. (1999b). Identifying stimuli of different perceptual categories in pure and mixed blocks of trials: Evidence for stimulus-driven switch costs. Acta Psychologica, 103, 173-205.

MAY R, U. (2001). Age differences in the selection of mental sets: The role of inhibition, stimulus ambiguity, and response-set overlap. Psychology \& Aging, 16, 96-109.

MaYr, U., \& KeELe, S. W. (2000). Changing internal constraints on action: The role of backward inhibition. Journal of Experimental Psychology: General, 129, 4-26.

Meiran, N. (1996). Reconfiguration of processing mode prior to task performance. Journal of Experimental Psychology: Learning, Memory, \& Cognition, 22, 1423-1442.
Meiran, N. (2000a). Modeling cognitive control in task-switching. Psychological Research, 63, 234-249.

MeIRAn, N. (2000b). Reconfiguration of stimulus task-sets and response task-sets during task-switching. In S. Monsell \& J. Driver (Eds.), Control of cognitive processes: Attention and performance XVIII ( pp. 377400). Cambridge, MA: MIT Press.

Meiran, N., Chorev, Z, \& SAPIR, A. (2000). Component processes in task switching. Cognitive Psychology, 41, 211-253.

Meiran, N., Hommel, B., Bibi, U., \& Lev, I. (2002). Consciousness and control in task switching. Consciousness \& Cognition, 11, 10-23.

Meiran, N., Neulinger, Y., \& May R, U. (2002). Cue-based differences in control strategies during task switching: Backward inhibition in perceptual classification. Manuscript in preparation.

MiLLER,J. (1978). Multidimensional same-different judgments: Evidence against independent comparisons of dimensions. Journal of Experimental Psychology: Human Perception \& Performance, 4, 411-422.

Miller, J., \& BAUER, D. W. (1981). Irrelevant differences in the "same""different" task. Journal of Experimental Psychology: Human Perception \& Performance, 7, 196-207.

Monsell, S., \& Driver J. (Eds.) (2000). Control of cognitive processes: Attention and performance XVIII. Cambridge, MA: MIT Press.

NAvon, D. (1977). Forest before trees: The precedence of global features in visual perception. Cognitive Psychology, 9, 353-383.

Norman, D. A., \& Shallice, T. (1986). Attention to action: Willed and automatic control of behavior. In R. J. Davidson, G. E. Schwartz, \& D. Shapiro (Eds.), Consciousness and self regulation (Vol 4, pp. 1-18). New York: Plenum.

Proctor, R. W., \& Fisicaro, S. A. (1977). Time, capacity, and selection between perceptual attributes. Journal of Experimental Psychology: Human Perception \& Performance, 2, 337-348.

Quinlan, P. T., \& HiLl, N. I. (1999). Sequential effects in rudimentary auditory and visual tasks. Perception \& Psychophysics, 61, 375-384.

Rogers, R. D., \& Monsell, S. (1995). The cost of a predictable switch between simple cognitive tasks. Journal of Experimental Psychology: General, 124, 207-231.

Santee, J. L., \& Egeth, H. E. (1980). Selective attention in the speeded classification and comparison of multidimensional stimuli. Perception \& Psychophysics, 28, 191-204.

SCHNEIDER, W. (1988). Micro Experimental Laboratory: An integrated system for IBM PC compatibles. Behavior Research Methods, Instruments, \& Computers, 20, 206-217.

Shalev, L., \& Algom, D. (2000). Stroop and Garner effects in and out of Posner's beam: Reconciling two conceptions of selective attention. Journal of Experimental Psychology: Human Perception \& Performance, 26, 997-1017.

Spector, A., \& Biederman, I. (1976). Mental set and mental shift revisited. American Journal of Psychology, 89, 669-679.

SPEnce, C., \& Driver, J. (1997). On measuring selective attention to an expected sensory modality. Perception \& Psychophysics, 59, 389-403.

VAn Duren, L. L., \& Sanders, A. F. (1988). On the robustness of the additive factors stage structure in blocked and mixed choice reaction designs. Acta Psychologica, 69, 83-94.

WARD, L. M. (1982). Determinants of attention to local and global features of visual forms. Journal of Experimental Psychology: Human Perception \& Performance, 8, 562-581.

Watanabe, T. (1988). Effect of irrelevant differences as a function of the relations between relevant and irrelevant dimensions in the samedifferent task. Journal of Experimental Psychology: Human Perception \& Performance, 14, 132-142.

(Manuscript received September 25, 2001; revision accepted for publication March 26, 2002.) 